Research Article

\title{
Coordinated Control for Driving Mode Switching of Hybrid Electric Vehicles
}

\author{
Yanyan Zuo, Rui Sun (D), Jiuyu Zang, and Mingyin Zheng \\ Institute of Noise and Vibration, Jiangsu University, Zhenjiang 212013, China \\ Correspondence should be addressed to Rui Sun; sunrui_ujs@163.com
}

Received 6 January 2020; Revised 25 June 2020; Accepted 2 September 2020; Published 15 September 2020

Academic Editor: Brij N. Agrawal

Copyright ( $\odot 2020$ Yanyan Zuo et al. This is an open access article distributed under the Creative Commons Attribution License, which permits unrestricted use, distribution, and reproduction in any medium, provided the original work is properly cited.

\begin{abstract}
Taking a hybrid electric vehicle using double-row planetary gear power coupling mechanism as a research object, this study proposes a coordinated control algorithm of "torque distribution, engine torque monitoring, and motor torque compensation" in an attempt to realize coordinated control for driving mode switching. Characteristic analysis of the power coupling mechanism was carried out, and the control strategy model in MATLAB/Simulink was built. Subsequently, the analysis of mode switching from the electric mode into joint driving mode was simulated. In addition, a multibody dynamics model of the power coupling mechanism was established and the simulation analysis during mode switching process was carried out. The results show that the proposed coordinated control strategy serves to effectively reduce the torque fluctuation and the impact degree during the mode switching process and improve the ride comfort of the vehicle. In the meantime, the time-domain and frequency-domain characteristics of gear meshing force and bearing restraint force indicate that the mode switching process of the dynamic coupling mechanism is quite stable and this control strategy contributes to improving the characteristics such as vibration and noise.
\end{abstract}

\section{Introduction}

Hybrid electric vehicles have become a research focus due to their low emissions and outstanding advantages in energy conservation, which has made them the best choice for lowering vehicle energy consumption [1]. The hybrid electric vehicle using the double-row planetary gear power coupling mechanism simplifies the structure of power train and overcomes the shortcomings of the single-row and threerow coupling systems. This configuration includes engine and motors and the hybrid electric vehicle has several working modes. The mode is frequently switched during the driving process. Since the dynamic response between the engine and motors is different, it may give rise to output torque fluctuation during the mode switching process [2]. In addition, the transient impact and vibration of the driving train affect ride comfort directly and necessitate an effective coordinated control $[3,4]$.

In order to solve this problem, some scholars have conducted relevant researches in this regard. Wang et al. proposed a motor torque compensation method based on
PID strategy to control the mode switching process of a parallel hybrid electric vehicle [5]. Moreover, Zhao et al. proposed a strategy based on engine torque estimation and motor torque compensation to control the mode switching process of a hybrid electric vehicle [6]. Liu et al. used the disturbance-free switching algorithm of the input redundant system to control the mode switching process [7]. Moreover, Parmar et al. adopted new test platform to test the vibration and noise of the mode switching of hybrid electric vehicle in an attempt to establish an appropriate coordinated control strategy to deal with the deterioration of the $\mathrm{NVH}$ [8].Tomura et al. controlled the motor to deal with the vibration caused by the mode switching $[9,10]$. Davis and Lorenz established an engine torque observer for ISG hybrid electric vehicles to calculate engine torque fluctuation and compensated torque by a motor to improve vehicle ride comfort [11]. Xiao et al. formulated the motor participated coordinated control for the mode switching, which improved the ride comfort of a two-speed electric vehicle [12]. Beck et al. used the Model Predictive Control (MPC) method to reduce the impact of the clutch engagement during 
parallel hybrid vehicle mode switching process, improved the ride comfort of switching from electric mode to joint driving mode, and verified the robustness of the control system [13]. Sun et al. used the MPC algorithm to control torque fluctuation during mode switching process and reduce impact effectively [14]. Koprubasi et al. adopted the optimal control theory to solve the target torques of vehicle power sources and reduce the impact during the mode switching process effectively [15].

Among all of the mode switching processes of hybrid electric vehicle, the engine starting process is the most unstable. First of all, there are many resistances in the engine starting process. These resistances will obviously slow down the engine starting speed. Then, temperature is the most important factor considering the external influence. When the engine is just started, the temperature is low and the viscosity of the lubricating oil is large, so the friction resistance between the components is large. At the same time, when the temperature is low, the atomization of fuel is poor, which leads to the deterioration of combustion process and the slow starting process of engine. When the working condition of the engine is stable, the output torque will be stable. It can be seen that the dynamic response of the engine is slow due to internal and external factors. In the mode switching process, the response difference between power sources will cause the fluctuation of the vehicle output torque, resulting in poor ride comfort. Therefore, it is necessary to develop a control strategy to compensate the engine to improve the vehicle ride comfort during mode switching process. The control technology of the motor is mature, which can realize the high-performance closed-loop control of the motor. Therefore, using the motor to compensate the engine is a simple and effective method.

In this paper, a hybrid electric vehicle using double-row planetary gear power coupling mechanism is selected as the research object. A dynamic coordinated control strategy of "torque distribution + engine torque monitoring + motor torque compensation" is proposed. The control strategy model was built in MATLAB/Simulink, and the vehicle model established in LMS.AMESim was used for the joint simulation. Furthermore, the rigid-flexible coupled dynamics model of the double-row planetary gear power coupling mechanism was established. We carried out the simulation analysis of the dynamics model during mode switching process in order to verify the proposed control strategy.

\section{System Structure}

As shown in Figure 1, the power coupling mechanism studied in this paper consists of double rows of planetary gears. The sun gear of the front row is connected to the motor MG1; the planet carrier of the front row is connected to the engine through the torsional vibration damper. The sun gear of the rear row is connected to the motor MG2 and the planet carrier is fixed, and the front and rear rows share the composite ring gear. Table 1 shows the main components and parameters of the hybrid electric vehicle.

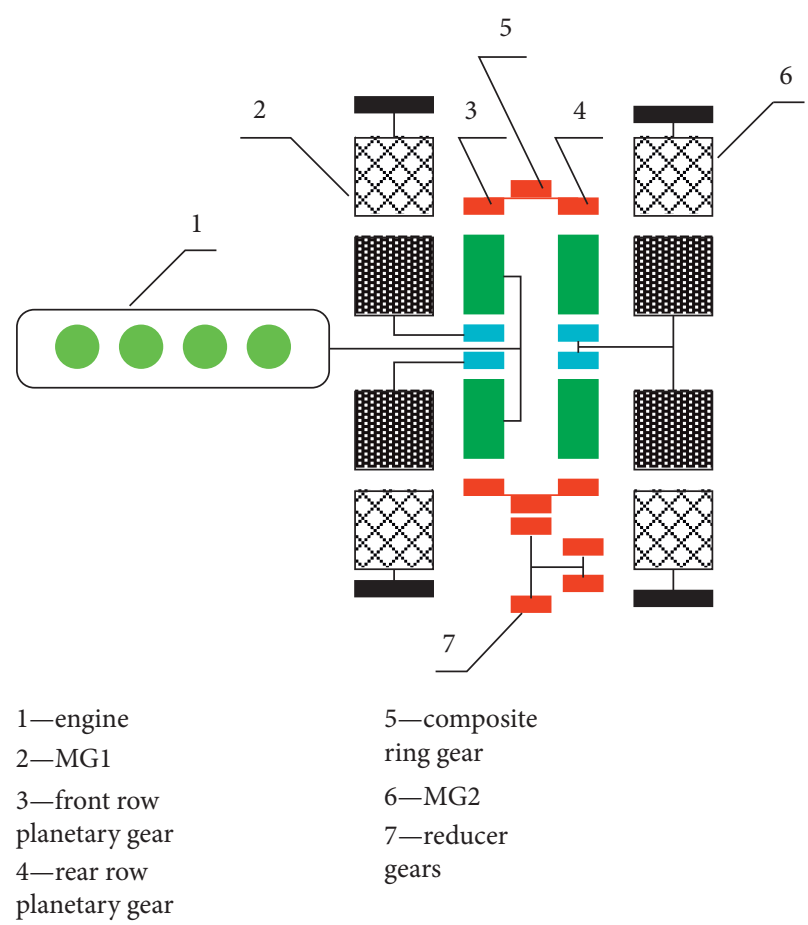

FIgURE 1: Double-row planetary gear power coupling system.

TABle 1: The main components and parameters of the hybrid electric vehicle.

\begin{tabular}{lcc}
\hline Component & Parameter & Value \\
\hline \multirow{2}{*}{ Engine } & Power (kW) & 73 \\
MG1 & Peak torque (Nm) & 142 \\
\multirow{2}{*}{ MG2 } & Power (kW) & 42 \\
& Power (kW) & 60 \\
Battery & Peak torque (Nm) & 207 \\
& Maximum power (kW) & 27 \\
Coupling mechanism & Battery capacity (Ah) & 6.5 \\
& Front row characteristic parameter & 2.6 \\
& Rear row characteristic parameter & 2.636 \\
\hline
\end{tabular}

Depending on the vehicle demand, the driving system flexibly combines the engine and the motors. When the vehicle runs in low power, it operates in electric mode while the engine is shut down to reduce emissions. When the vehicle runs in a normal state, the system keeps the engine in an optimal working condition, and in the meantime the battery can be charged by the motor MG1. As a result, the system can greatly improve the vehicle energy efficiency and the fuel economy [16]. Due to the large difference in dynamic response between the engine and the motors, it may give rise to output torque fluctuation during the mode switching process. In addition, the shock and vibration and noise of the driving train directly affect ride comfort and necessitate a coordinated control.

\section{Dynamic Coordination Control Strategy}

3.1. Analysis of the Characteristics of the Power Coupling Mechanism. In order to analyze the mechanism, a diagram 
is used to indicate its power transmission, as shown in Figure 2.

The power coupling mechanism is analyzed using the isolation method. The engine and front row planet carrier are considered together and the dynamics equation can be expressed in accordance to

$$
T_{\mathrm{e}}-T_{\mathrm{cl}}=\dot{\omega} \mathrm{e} \cdot J_{\mathrm{e}},
$$

where $T_{\mathrm{e}}$ denotes the output torque of the engine, $T_{\mathrm{cl}}$ denotes the internal torque of the front row planet carrier, $\omega_{\mathrm{e}}$ denotes angular velocity of the engine, and $J_{\mathrm{e}}$ denotes the sum of inertia moments of the engine crankshaft, the output shaft, and the front row planet carrier.

Analyzing the motor MG1 and front row sun gear, the following equation can be obtained:

$$
T_{\mathrm{MG} 1}-T_{\mathrm{s} 1}=\dot{\omega}_{\mathrm{MG} 1} \cdot J_{\mathrm{MG} 1},
$$

where $T_{\mathrm{MG} 1}$ denotes output torque of the motor MG1, $T_{\mathrm{s} 1}$ denotes the internal torque of the front row sun gear, $\omega_{\mathrm{MG} 1}$ is the angular velocity of motor MG1, and $J_{\mathrm{MG}}$ denotes the sum of the inertia moments of the front row sun gear and the MG1 rotor.

Analyzing motor MG2 and rear row sun gear, the following equation can be obtained:

$$
T_{\mathrm{MG} 2}-T_{\mathrm{s} 2}=\dot{\omega}_{\mathrm{MG} 2} \cdot J_{\mathrm{MG} 2},
$$

where $T_{\mathrm{MG} 2}$ denotes output torque of the MG2, $T_{\mathrm{s} 2}$ denotes the internal torque of the rear row sun gear, $\omega_{\mathrm{MG} 2}$ is the angular velocity of the MG2, and $J_{\mathrm{MG} 2}$ denotes the sum of the inertia moments of the rear row sun gear and the MG2 rotor.

The dynamics equation of the composite ring gear can be expressed in accordance to

$$
T_{\mathrm{r} 1}+T_{\mathrm{r} 2}-T_{\text {com }}=\dot{\omega}_{\text {com }} \cdot J_{\text {com }},
$$

where $T_{\mathrm{r} 1}$ and $T_{\mathrm{r} 2}$ are the internal torque of the front and rear rows ring gears, respectively, $T_{\text {com }}$ denotes the torque of composite ring gear, $\omega_{\text {com }}$ denotes the angular velocity of composite ring gear, and $J_{\text {com }}$ denotes the inertia moment of the composite ring gear.

The transmission characteristics of planetary gears can be expressed in accordance to

$$
\begin{aligned}
\left(1+k_{1}\right) \omega_{\mathrm{e}} & =\omega_{\mathrm{MG} 1}+\frac{k_{1}}{k_{2}} \omega_{\mathrm{MG} 2}, \\
T_{\mathrm{s} 1} & =\frac{T_{\mathrm{c} 1}}{1+k_{1}}=\frac{T_{\mathrm{r} 1}}{k_{1}}, \\
T_{\mathrm{r} 2} & =k_{2} T_{\mathrm{s} 2},
\end{aligned}
$$

where $k_{1}$ and $k_{2}$ denote the characteristic parameters of the front and rear rows planetary gears.

When the power coupling mechanism is in different working modes, the mathematical model will evolve into different forms.

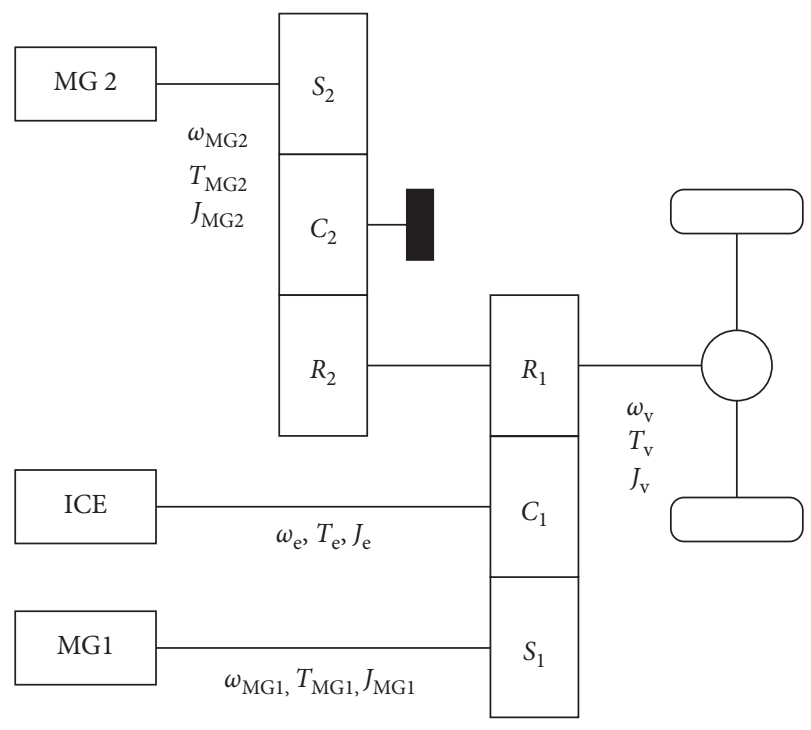

Figure 2: Power transmission of coupling mechanism.

3.2. Engine Torque Estimation. The key of coordinated control for mode switching is to control the real-time output torque of power sources, and then the total torque does not fluctuate greatly. The premise of this method is that the control system can obtain the real-time output torque of the engine. There are two ways to realize this function, one method is to use torque sensor to measure directly, and the other is to use algorithm to estimate engine torque indirectly. This paper uses BP neural network optimized by genetic algorithm to estimate engine torque.

\subsubsection{BP Neural Network Optimized by Genetic Algorithm.} BP neural network is composed of three parts: input layer, intermediate layer, and output layer. The intermediate layer can be expanded to multilayers. All neurons in the adjacent layers are connected, but there is no connection between neurons in the same layer. The main advantage of BP neural network is its strong nonlinear mapping ability. The structure of BP network model is as shown in Figure 3.

Genetic algorithm simulates the evolution theory and gene genetics principle in the development of nature and uses the continuous evolution to search the global optimal solution. The new species are more adaptable to the environment with each evolution, and the optimal individual in the last population is the optimal approximate solution.

3.2.2. Data Processing. Data processing is to transform the original data into that which can be recognized by neural network algorithm, and it is an important step of algorithm operation. Data processing can improve the data quality and plays an important role in improving the accuracy and performance of network training. The experimental data used in this paper are shown in Table 2. In Table 2, $\mathrm{n}(\mathrm{r} / \mathrm{min})$ denotes the speed, $T_{\mathrm{e}}(\mathrm{Nm})$ denotes the engine torque, and $\alpha$ (\%) denotes the throttle opening.

The data processing of neural network is the data normalization that is to scale the original data according to 


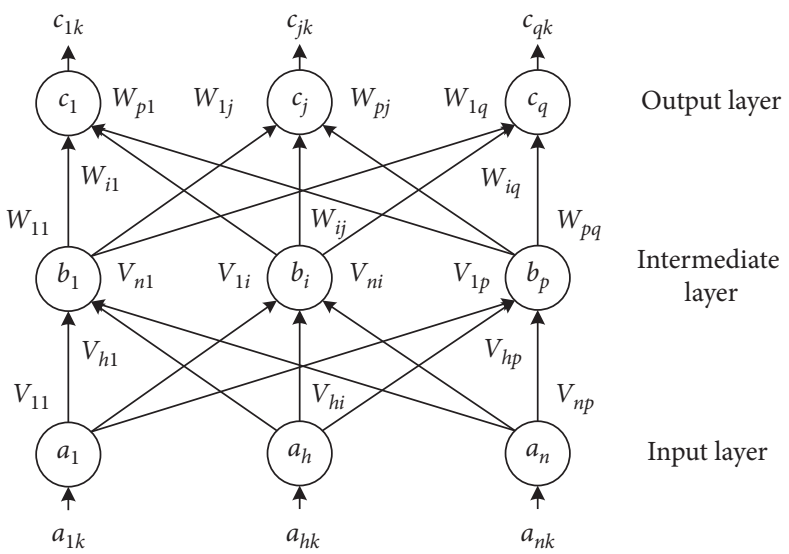

FIgURE 3: Structure of BP network model.

TABLe 2: Experimental data of engine.

\begin{tabular}{lcccccccccc}
\hline $\mathrm{n}^{\alpha} T_{\mathrm{e}} \alpha \alpha \alpha \alpha \alpha \alpha \alpha \alpha \alpha \alpha \alpha \alpha \alpha$ & 10 & 20 & 30 & 40 & 50 & 60 & 70 & 80 & 90 & 100 \\
\hline 800 & 25.4 & 61.2 & 83.7 & 101.4 & 103.3 & 104.8 & 105.3 & 105.8 & 106.5 & 107.4 \\
1000 & 22.8 & 54.9 & 79.5 & 100.8 & 104.6 & 105.1 & 105.4 & 106.2 & 107.8 & 108.5 \\
1200 & 20.2 & 49.6 & 76.2 & 99.7 & 107.3 & 108.6 & 109.1 & 109.9 & 110.2 & 111.6 \\
1400 & 16.3 & 45.5 & 72.4 & 98.4 & 108.4 & 110.9 & 111.5 & 112.5 & 113.8 & 114.3 \\
1600 & & 41.4 & 68.2 & 97.6 & 110.6 & 113.4 & 114.2 & 114.9 & 115.6 & 117.1 \\
1800 & & 37.2 & 64.1 & 95.4 & 113.1 & 115.8 & 116.7 & 117.1 & 118.4 & 120.2 \\
2000 & & 34.3 & 59.8 & 93.1 & 115.6 & 118.1 & 119.2 & 120.1 & 121.6 & 122.9 \\
2200 & & 31.1 & 55.7 & 90.8 & 117.5 & 122.8 & 123.8 & 124.7 & 125.3 & 126.8 \\
2400 & & 28.9 & 51.6 & 88.4 & 119.8 & 125.5 & 127.4 & 129.8 & 130.6 & 131.8 \\
2600 & & 23.5 & 47.9 & 87.6 & 120.8 & 127.2 & 130.8 & 132.6 & 133.5 & 134.6 \\
2800 & & 21.6 & 43.3 & 85.4 & 120.5 & 129.7 & 133.3 & 135.2 & 135.9 & 136.1 \\
3000 & & 19.6 & 40.1 & 82.1 & 120 & 130.3 & 135.1 & 136.6 & 137 & 137.5 \\
3200 & & & 37.5 & 78.9 & 117.9 & 131.1 & 136.4 & 137.5 & 138.1 & 138.4 \\
3400 & & & 34.9 & 76.5 & 115.6 & 132.2 & 137.9 & 138.2 & 138.9 & 139.6 \\
3600 & & & 31.6 & 72.8 & 113.5 & 133.3 & 138.3 & 138.9 & 139.8 & 140.8 \\
3800 & & & 28.2 & 69.2 & 112.6 & 134 & 139 & 139.2 & 140 & 141.9 \\
4000 & & 25.5 & 65.8 & 111.3 & 133.6 & 137.9 & 138.7 & 140.2 & 142.8 \\
4200 & & & 62.5 & 110.6 & 132.6 & 136.5 & 140.1 & 141 \\
4400 & & & 59.9 & 109.8 & 131.7 & 135.2 & 138.9 & 139.5 & 141.6 \\
4600 & & & 57.7 & 108.1 & 129.6 & 134.7 & 137.6 & 138.4 & 139 \\
4800 & & & 56.1 & 107.8 & 126.9 & 132.6 & 136 & 137.1 & 137.8 \\
5000 & & & 55.2 & 106.7 & 125.5 & 130.5 & 132.2 & 135 & 136.5 \\
5200 & & & & 105.9 & 124 & 128.6 & 129.5 & 134.6 & 135.5 \\
\hline & & & & & & & & &
\end{tabular}

specific rules, so that the processed data falls in a specific range. The calculation method is as follows:

$$
v_{i}^{\prime}=\frac{v_{i}-\min _{A}}{\max _{A}-\min _{A}}\left(\text { new_max } \max _{A}-\text { new_min }_{A}\right)+\text { new_min } \min _{A},
$$

where $v_{i}$ denotes the original data and $\max _{A}$ and $\min _{A}$ denote the maximum and minimum data.

3.2.3. BP Neural Network Model Training. The structure and parameters of BP neural network are shown in Table 3. The genetic algorithm error is shown in Figure 4.

Using genetic algorithm is to find the optimal weight and threshold value and assign these values to BP neural network for training. Figure 5 shows the error between the BP neural network training results and the samples.

Using the nonlinear network obtained by the above algorithm, the output torque estimation of engine is carried out, and the map of engine torque estimation is obtained, as shown in Figure 6.

3.3. Coordinated Control Strategy for the Mode Switching Process. In essence, the driving torque fluctuation and longitudinal impact during mode switching are attributed to the difference in the dynamic response of the engine and motors. Therefore, the process of "engine starting or stopping" necessitates coordinated control. On this basis, this paper proposed a dynamic coordinated control strategy of "torque distribution + engine torque monitoring + motor 
TABLE 3: Structure and parameters of algorithm.

\begin{tabular}{|c|c|c|}
\hline Algorithm & & Parameters \\
\hline \multirow{11}{*}{ BP neural network } & Number of neurons in input layer & 2 \\
\hline & Number of neurons in the output layer & 1 \\
\hline & Number of intermediate layers & 1 \\
\hline & Number of neurons in the intermediate layer & 9 \\
\hline & Network structure & $2 \times 9 \times 1$ \\
\hline & Input-intermediate layer transfer function & Tansig \\
\hline & Intermediate-output layer transfer function & Logsig \\
\hline & Training algorithm & Trainlm \\
\hline & Maximum training times & 1000 \\
\hline & Learning rate & 0.1 \\
\hline & MSE & 0.001 \\
\hline \multirow{5}{*}{ Genetic algorithm } & Initial population size & 50 \\
\hline & Code binary digit & 10 \\
\hline & Genetic algebra & 100 \\
\hline & Crossover probability & 0.7 \\
\hline & Mutation probability & 0.01 \\
\hline
\end{tabular}

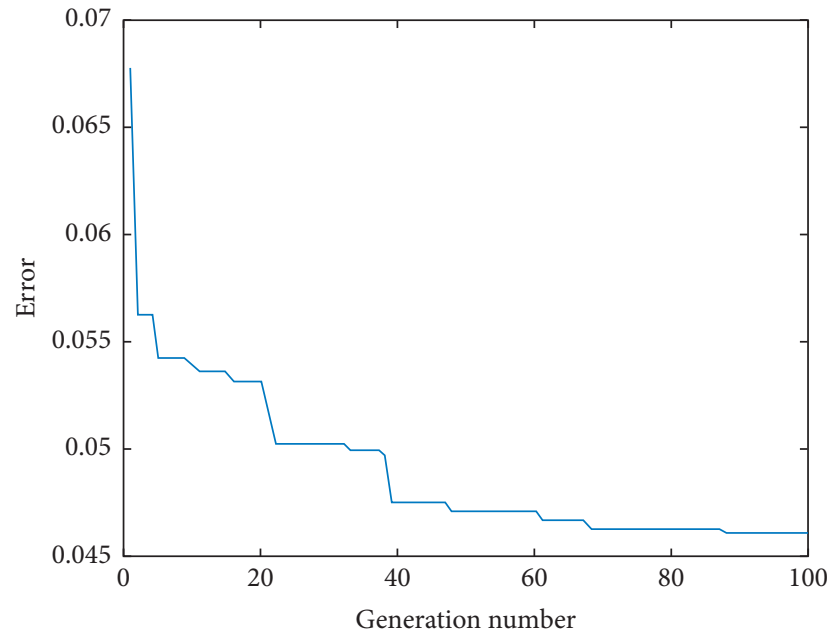

FIgURE 4: Genetic algorithm error.

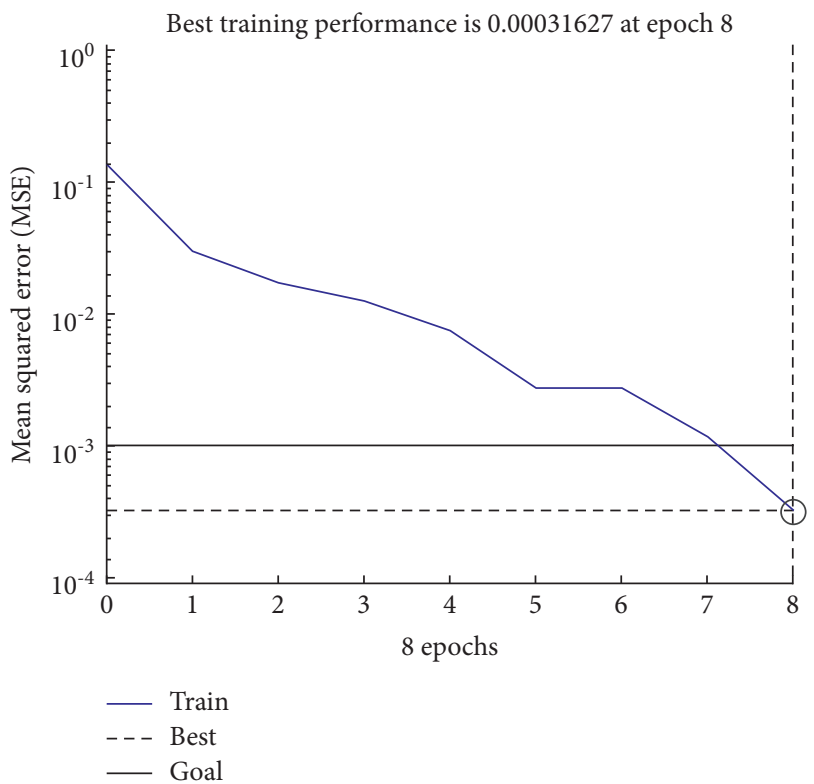

FIGURE 5: BP neural network error.

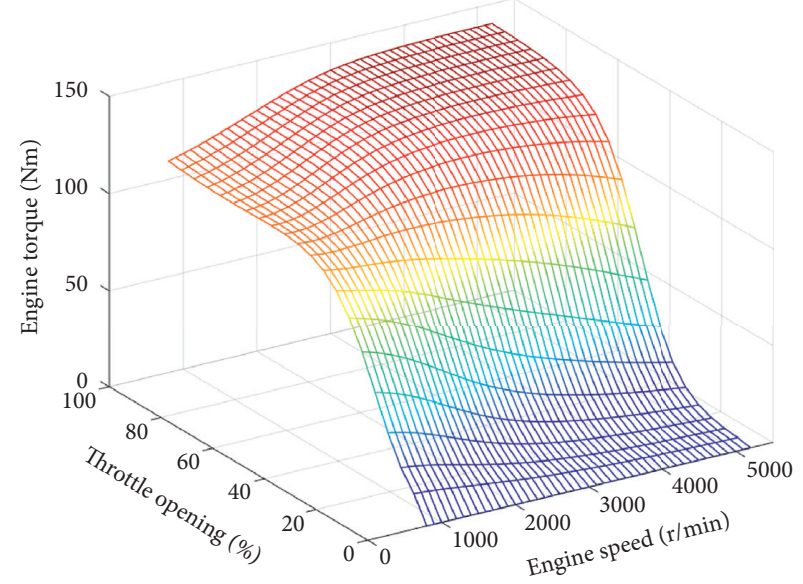

Figure 6: MAP of engine torque estimation.

torque compensation" to improve the ride comfort during the mode switching process. First, the engine torque and the motor torque are distributed according to the torque demand (as shown in (9)), and then the engine state is monitored to determine whether or not to perform coordinated control (as shown in (10)). After entering the coordinated control, the motor compensates the engine torque to reduce the difference between the actual output torque and demand torque (as shown in (11)). Finally, the coordinated control is stopped when the value of the engine output torque is in the demand range (as shown in (12)):

$$
\begin{aligned}
\left(T_{\text {e_req }}, T_{\text {MG1_req }}, T_{\text {MG2_req }}\right) & =f\left(T_{\text {req }}\right), \\
\text { Switch_flag_start } & =f\left(T_{\text {e_req }}\right), \\
\left(T_{\text {MG1_real }}, T_{\text {MG2_real }}\right) & =f\left(T_{\text {e_real }}, k_{1}, k_{2}\right), \\
\text { Switch_flag_stop } & =f\left(T_{\text {e_req }}, T_{\text {e_real }}\right),
\end{aligned}
$$

where $T_{\text {req }}, T_{\text {e_req }}, T_{\text {MG1_req }}$, and $T_{\text {MG2_req }}$ denote the necessary torques of the whole vehicle, engine, motor MG1, and 
motor MG2, respectively; $T_{\text {e_real }}, T_{\text {MG1_real }}$, and $T_{\text {MG2_real }}$ denote the actual torques of the engine, motor MG1, and motor MG2, respectively; and Switch_flag_start and Switch_flag_stop denote the start and stop identification of the coordination control strategy.

Subsequently, the switching process from electric mode to the joint driving mode is taken as an example. The lever method is used to analyze the working condition of the system at this stage, as shown in Figure 7. When the engine is started in electric mode, the motor MG1 will drive the engine to rotate to the starting speed, and the engine will be started thereafter. The power provided by the engine is divided into two parts by the front row planetary gear. Concisely, the one part transmitted to the composite ring gear is employed to drive the vehicle, and the other part transmitted to the front row sun gear is used to drive the motor MG1 to generate electricity. According to the actual demand of the vehicle, the electric energy is consumed to drive the vehicle or charge the battery. Figure 8 is the diagram of power transmission path to drive the engine by the motor MG1 in the pure electric mode.

The flow chart of the dynamic coordination control during this mode switching process is shown in Figure 9.

When the vehicle operates in the pure electric mode, it is driven by the motor MG2, the engine, and motor MG1 which are stopped, so $T_{\mathrm{e}}=T_{\mathrm{MG} 1}=0, \omega_{\mathrm{e}}=\omega_{\mathrm{c} 2}=0$, and the output torque can be expressed in accordance to

$$
T_{\text {out }}=k_{2} T_{\mathrm{MG} 2} \text {. }
$$

When the engine is started based on the torque distribution strategy, the start flag of the dynamic control strategy Switch_flag_start is set, and the torque compensation is started by motor MG2. The compensation torque can be expressed in accordance to

$$
T_{\text {MG2_add }}=\left(T_{\text {e_req }}-T_{\text {e_real }}\right) \frac{k_{1}}{\left(1+k_{1}\right) k_{2}} .
$$

Therefore, the actual torque demand of the motor MG2 can be expressed as

$$
T_{\text {MG2_total }}=T_{\text {MG2_req }}+T_{\text {MG2_add }} \text {. }
$$

The motor MG2 keeps torque compensation during the process of the engine starting up. When the actual torque of the engine reaches $95 \%$ of the necessary torque, the torque compensation can be considered to be ended. In this case, the stop flag Switch_flag_stop is set, while the torque compensation stops.

In the joint driving mode, when the engine is working, its output power is divided into two parts through the front row planetary gear: one part drives the vehicle through the ring gear, and the other part drives the motor MG1 to generate electricity, and the output torque can be expressed as

$$
T_{\text {out }}=T_{\mathrm{e}} \cdot \frac{k_{1}}{1+k_{1}}+k_{2} T_{\mathrm{MG} 2} .
$$

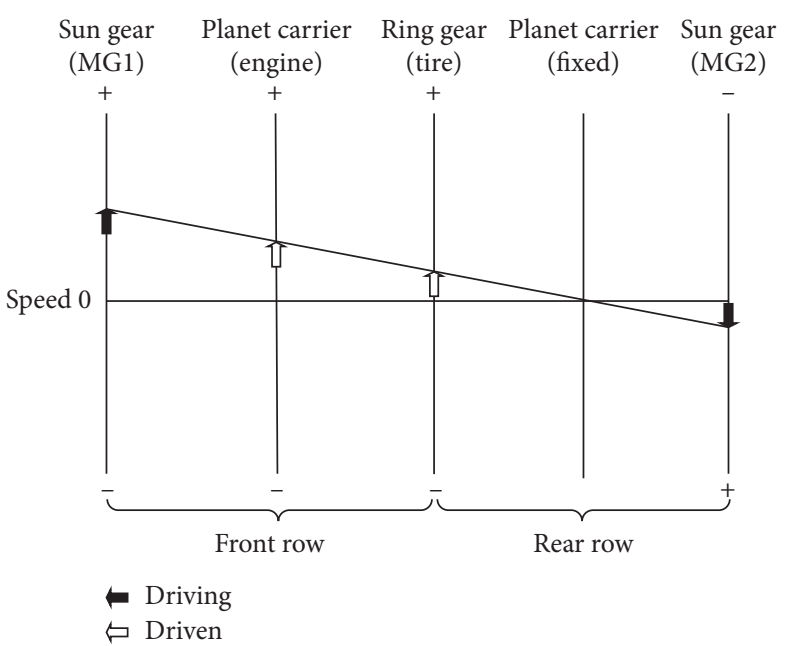

FIGURE 7: Lever method of speed.

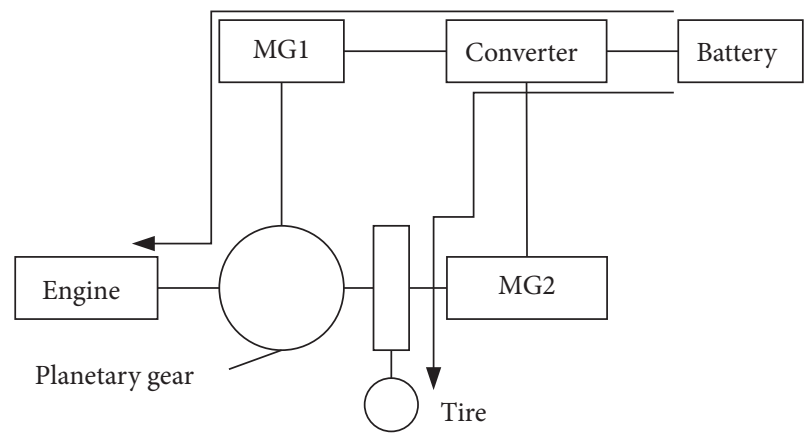

Figure 8: Power transmission of engine starting process.

\section{Simulation}

There are subjective and objective evaluation methods for ride comfort evaluation. Subjective evaluation method varies from person to person due to personal feelings. Therefore, the objective evaluation method can be used to make more accurate judgments. In this case, the concept of impact degree can be introduced as the evaluation index of the mode switching process. The impact degree is defined as the changing rate of the longitudinal acceleration of the vehicle [17], which can be expressed as in the following equation. The smaller the value of the impact degree, the smoother the mode switching process is [18]

$$
J=\frac{\mathrm{d} a}{\mathrm{~d} t}=\frac{\mathrm{d}^{2} v}{\mathrm{~d} t^{2}}
$$

In addition, the characteristics of vibration and noise of the power coupling mechanism are closely related to the meshing transmission of the planetary gears [19]. Moreover, the bearing restraint force is the excitation of the mechanism, which directly affects the noise of the power coupling mechanism. In this paper, the dynamic simulation was carried out using the model of the power coupling mechanism. The time-domain and frequency-domain characteristics of the gear meshing force and the bearing restraint 


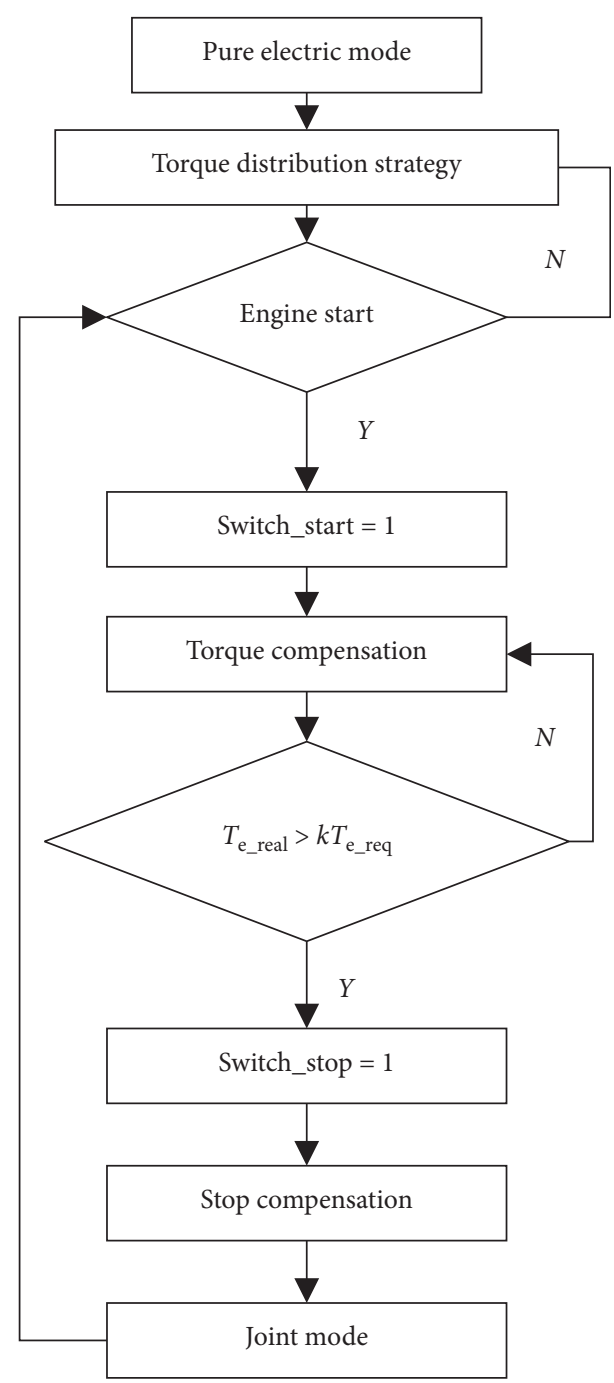

FIGURE 9: Flow chart of dynamic coordinated control.

force were analyzed to delve into the vibration and noise characteristics of the mode switching.

4.1. One-Dimensional Dynamics Simulation. With the LMS.AMESim, a vehicle dynamics model was established. The models of vehicle energy distribution strategy and coordinated control strategy for the mode switching were established in MATLAB/Simulink; thus, joint simulation was carried out. The results are shown in Figures 10-12.

The dynamic model was simulated using the New European Driving Cycle (NEDC) [20]. The speed following situation is shown in Figure 10. It can be seen from the figure that the speed of the vehicle is well controlled under this condition, which demonstrates that its power performance meets fully the demand.

Figure 11(a) shows the change of output torque of each power source at the switching moment when there is no coordinated control strategy. It can be seen from the figure that power source is controlled according to the target torque during mode switching and the motor responds quickly, while the engine responds slowly and the control

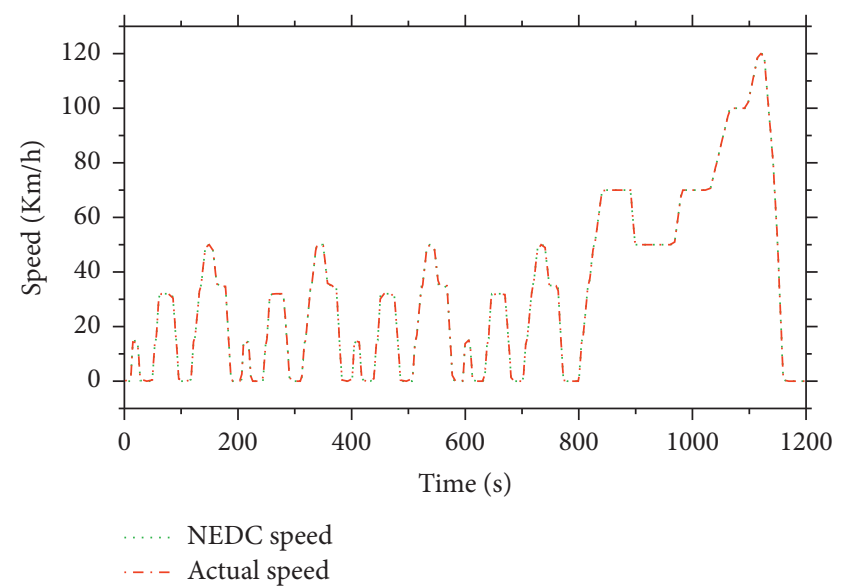

FIGURE 10: NEDC simulation result.

target cannot be reached quickly. Figure 11(b) demonstrates the torque variation of each power source after applying the coordinated control strategy. It can be seen that the motor MG2 does not immediately change to the target torque at the switching moment; instead, it performs torque compensation according to the engine torque change in an attempt to ensure the low fluctuations of the total output torque. Figure 10 shows the vehicle impact degree before and after control. It can be seen that the impact degree $J$ before control is more than $9 \mathrm{~m} / \mathrm{s}^{3}$ and that after control it is less than $1 \mathrm{~m} /$ $s^{3}$, as shown in Table 4 . As a result, the vehicle longitudinal impact is greatly reduced, and the ride comfort is much improved.

In summary, after the coordinated control strategy was added to the vehicle, the maximum torque deviation and the maximum impact degree were greatly reduced; the vehicle ride comfort is improved.

4.2. 3D Solid Model Dynamics Simulation. In order to study the vibration and noise at the switching moment, the solid model of the whole system was established. Then, it was imported into ADAMS for constructing a multirigid dynamic model. Thereafter, ANSYS was performed to flex the input shaft of the motors and engine. The rigid-flexible coupled dynamics model was established finally, as shown in Figure 13.

In order to study the dynamic characteristics of the power coupling mechanism under this control strategy, the analysis of mode switching was performed. The simulation load is the torque converted from whole vehicle to the ring gear and power sources data obtained by the joint simulation. The results are as follows.

4.2.1. Characteristics of the Angular Velocity. Figure 14 shows the shafts angular velocity of the motor MG1 and the engine, respectively. It can be seen from Figure 14(b) that the speed of the engine increases from $0 \mathrm{deg} / \mathrm{s}$ to the idle speed of $4800 \mathrm{deg} / \mathrm{s}$ at $0.25 \mathrm{~s}$; then the engine is ignited to output torque. The rotation speed is equivalent to the theoretical value, which verifies the accuracy of the simulation. 


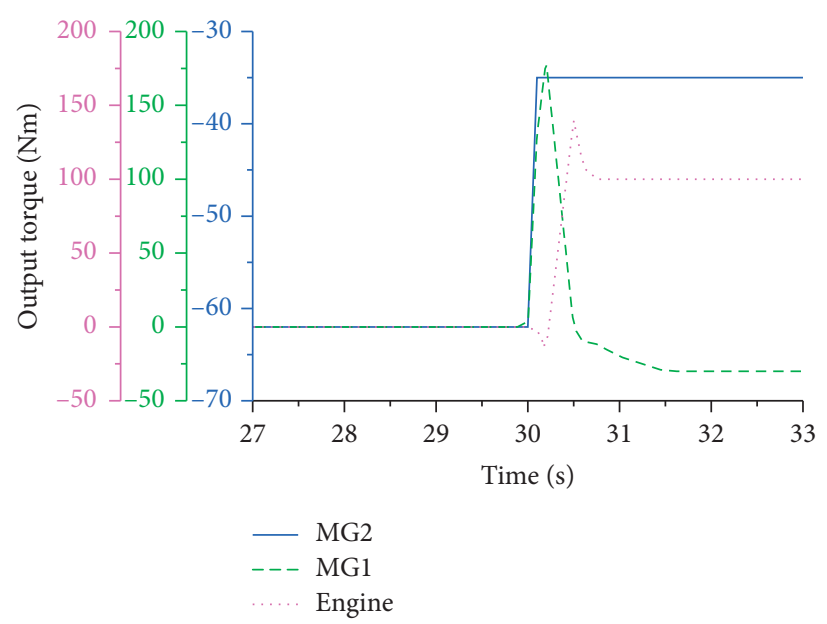

(a)

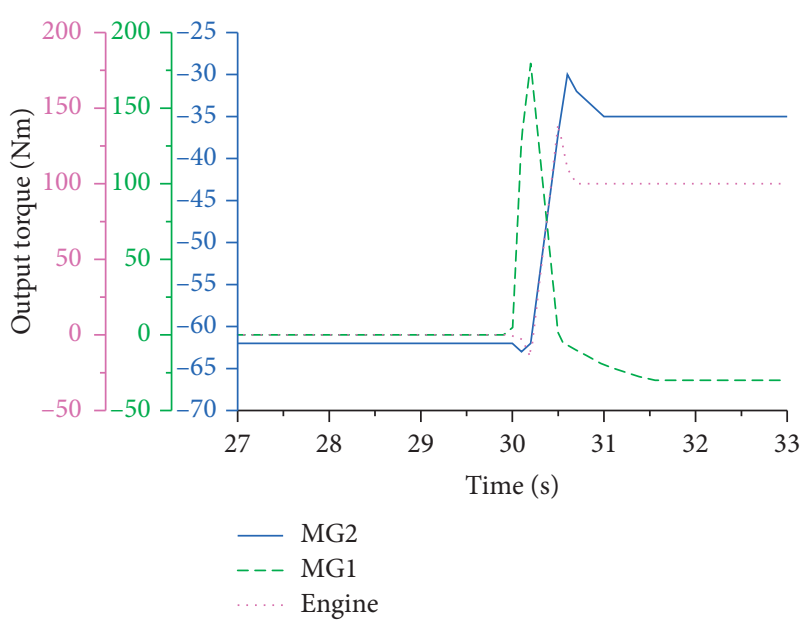

(b)

FIGURE 11: Output torque of power sources. (a) Output torque of each power source before control. (b) Output torque of each power source after control.

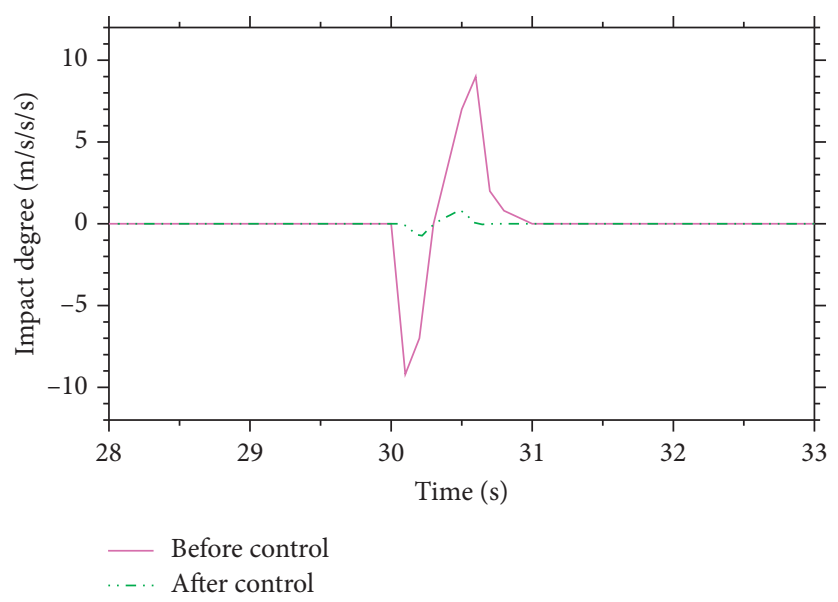

FIGURE 12: Comparison of impact degree before and after control.

TABLE 4: Comparison before and after coordinated control.

\begin{tabular}{lccr}
\hline & Before control & After control & Improvement percentage \\
\hline Maximum torque deviation $(\mathrm{Nm})$ & 264.11 & 48.3 & 81.71 \\
Maximum impact degree $\left(\mathrm{m} / \mathrm{s}^{3}\right)$ & 9.28 & 0.94 & 89.87 \\
\hline
\end{tabular}

4.2.2. Characteristics of the Meshing Force. The vibration and noise of the power coupling mechanism are closely associated with the meshing of the planetary gears. Therefore, the meshing force between the gears is selected as the research object. In the analysis of the meshing force characteristics of the planetary gears, the meshing frequency can be expressed in accordance to the following equation [21]:

$$
f=\frac{\left(n_{\mathrm{p}}-n_{\mathrm{c}}\right) z_{\mathrm{p}}}{60}=\frac{\left(n_{\mathrm{s}}-n_{\mathrm{c}}\right) z_{\mathrm{s}}}{60}=\frac{\left(n_{\mathrm{r}}-n_{\mathrm{c}}\right) z_{\mathrm{r}}}{60},
$$

where $n_{\mathrm{s}}, n_{\mathrm{p}}, n_{\mathrm{c}}$, and $n_{\mathrm{r}}$ denote the rotational speed of the sun gear, planet gear, planet carrier, and ring gear in the planetary gears, respectively; Likewise, $z_{\mathrm{s}}, z_{\mathrm{p}}$, and $z_{\mathrm{r}}$ denote the teeth number of the sun gears, planet gears, and ring gears of the planetary gears, respectively.

The meshing frequency of rear row can be solved with (18). In addition, the front row works unstably during the engine starting process, so there is no fixed meshing frequency.

Figure 15 shows the time-domain and frequency-domain characteristics of the meshing force. The meshing force of the front row changes during the mode switching process. After the engine outputs torque, the gear meshing force increases steadily, and no prominent peak is seen in the 


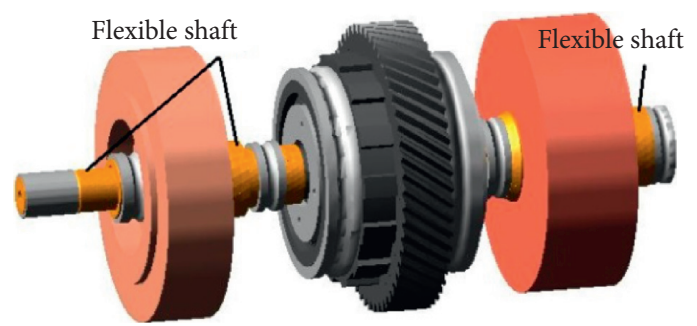

Figure 13: The rigid-flexible coupled dynamics model.

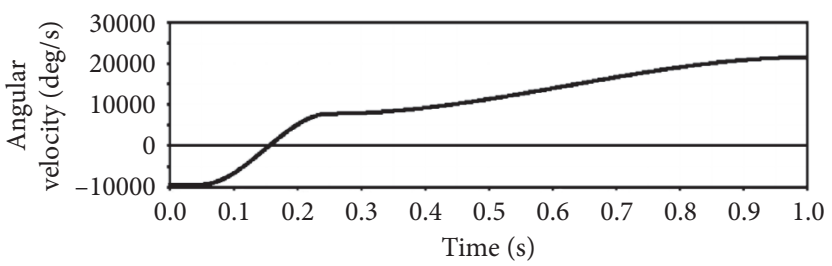

(a)

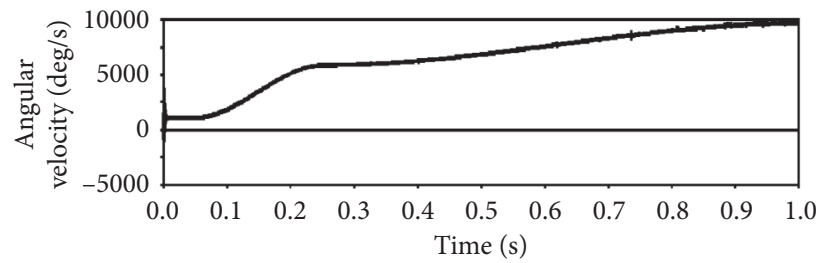

(b)

FIgURe 14: Angular velocity of shafts. (a) Angular velocity of the shaft of motor MG1. (b) Angular velocity of the engine shaft.
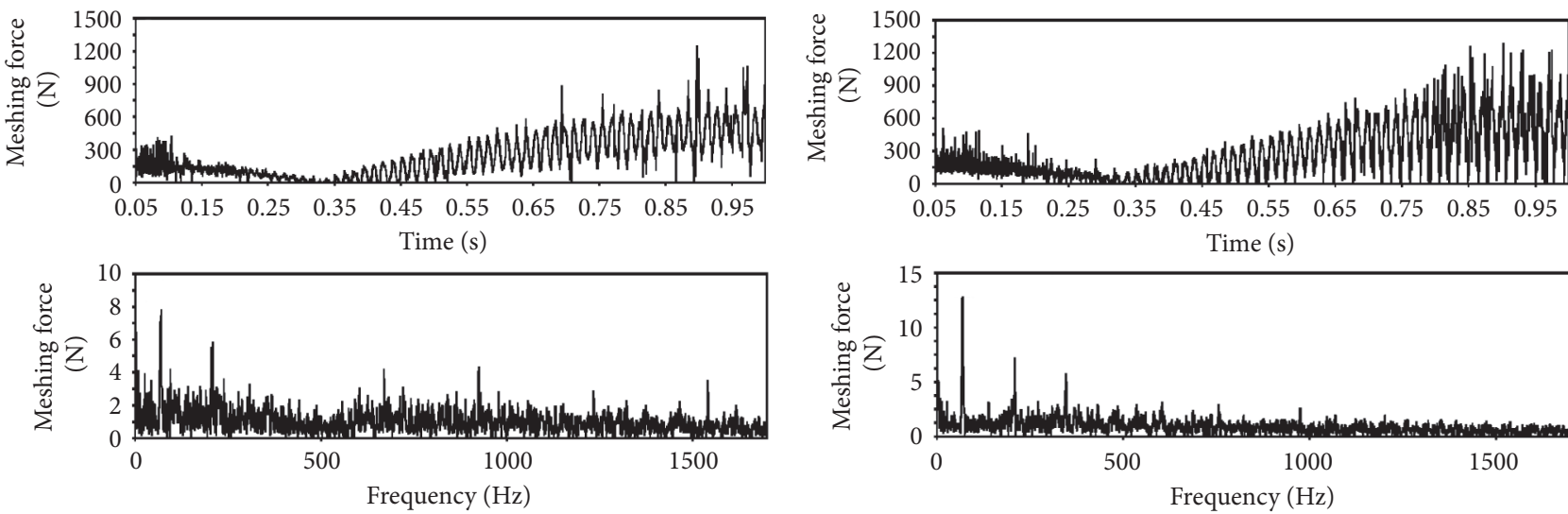

(a)

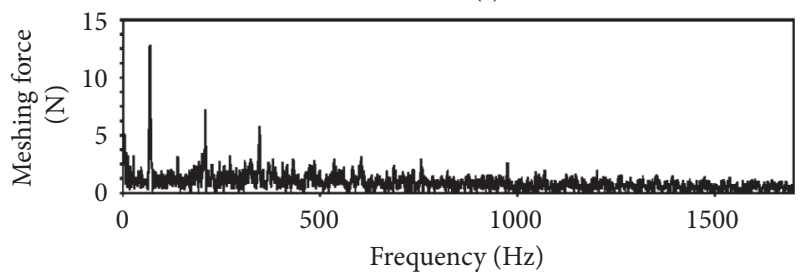

(b)
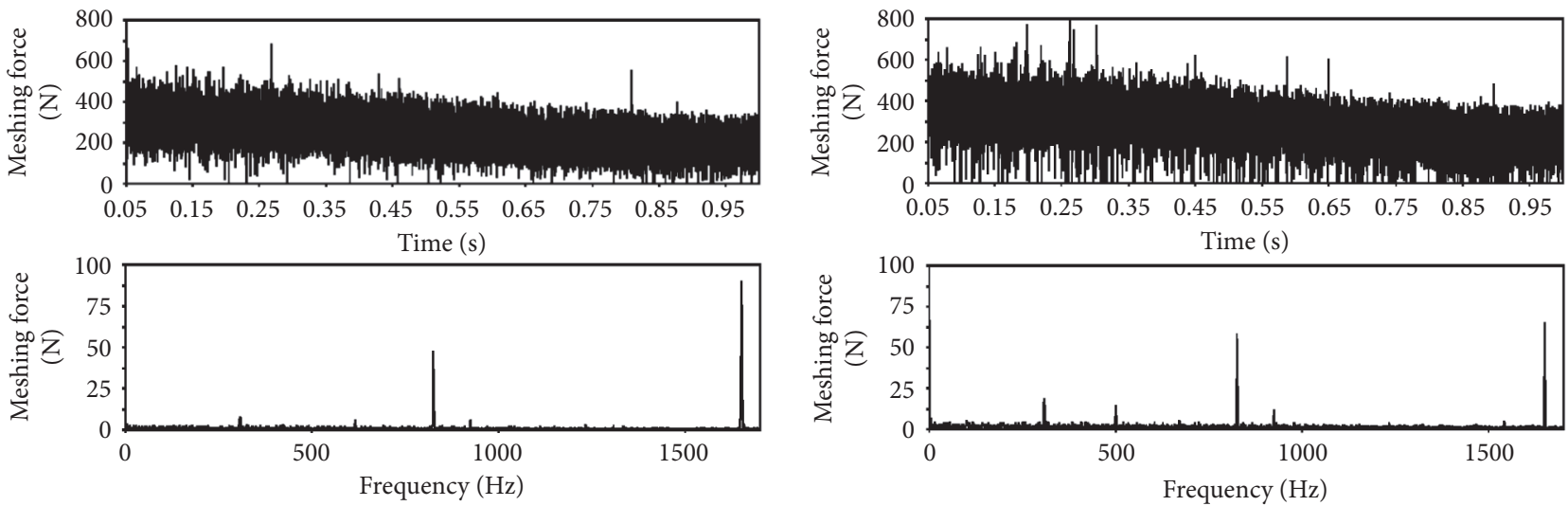

(c)

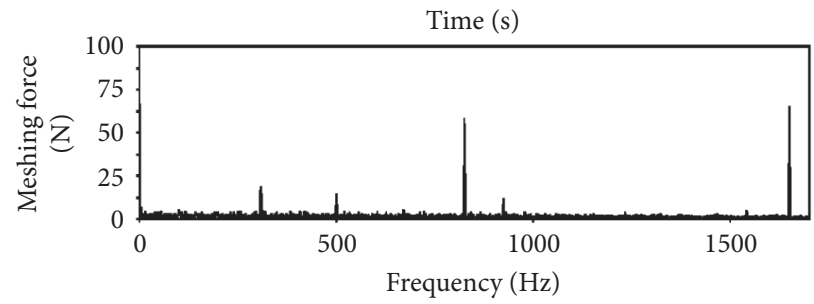

(d)

FIGURE 15: The time-frequency characteristics of the meshing force. (a) Sun gear and planet gear of front row. (b) Ring gear and planet gear of front row. (c) Sun gear and planet gear of rear row. (d) Ring gear and planet gear of rear row. 

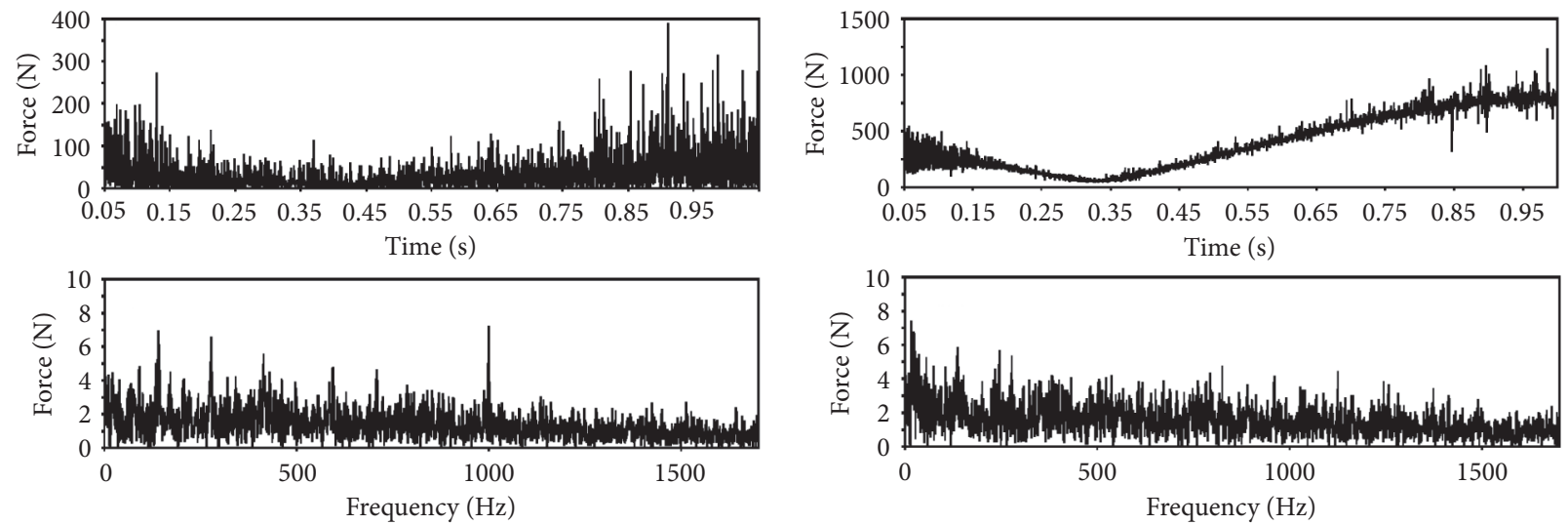

(a)

(b)
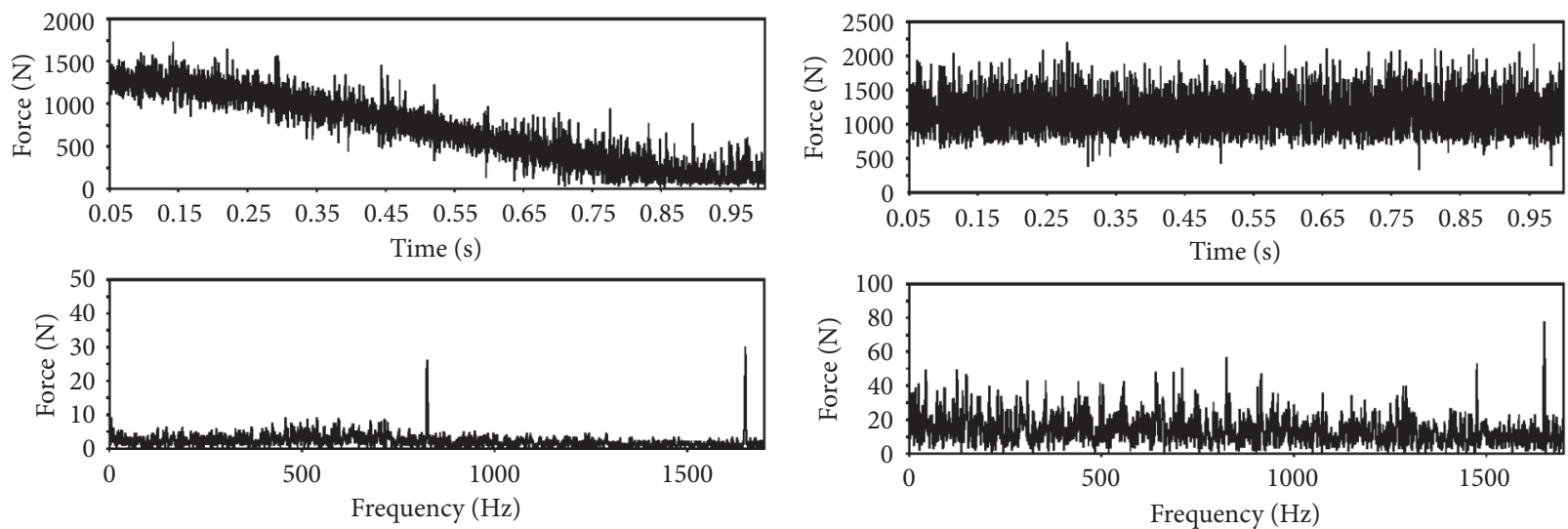

(c)

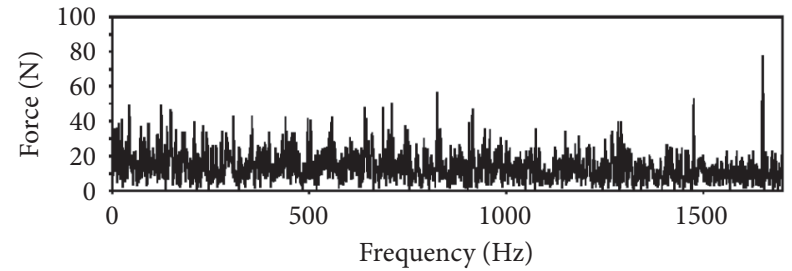

(d)

FIGURE 16: The time-domain and frequency-domain characteristics of the bearing restraint force. (a) Input shaft bearing of engine. (b) Input shaft bearing of MG1. (c) Input shaft bearing of MG2. (d) Bearing of composite ring gear.

frequency characteristics. The meshing force of rear row also decreases steadily along with the MG2 output torque, and a prominent peak is seen at its meshing frequency $f_{\mathrm{R}}=825 \mathrm{~Hz}$ and its double meshing frequency $f_{\mathrm{R}}=1650 \mathrm{~Hz}$.

4.2.3. Characteristics of Bearing Restraint Force. The bearing restraint force acts as an excitation source to induce the power coupling mechanism to generate vibration, which has important significance in terms of analysis of the vibration and noise characteristics of the power coupling mechanism. In this paper, the rotating pair is set in each bearing center to simulate the bearing, and the time-domain and frequencydomain characteristics of the bearing restraint force were observed, as shown in Figure 16.

According to Figure 16, the time-domain and frequencydomain characteristics of the bearing restraint force show the following:

(1) During the mode switching, the engine is driven by the motor MG1 to obtain the idle speed and starts to output torque. The time-domain characteristics of bearing restraint force of motor MG1 and engine change smoothly during this process. In addition, the frequency-domain characteristics remain stable without obvious peak values.
(2) The bearing restraint force of the input shaft of motor MG2 has peaks at its meshing frequency $f=$ $825 \mathrm{~Hz}$ and $f=1650 \mathrm{~Hz}$. The engine starting process has a small impact on the rear bearing. However, owing to the torque compensation strategy of the motor, the rear row operates stably.

(3) The composite ring gear couples the torque of the engine and the motors. During the switching process, it must undertake the impact of the engine. As a result, its dynamic characteristics are more complicated, but it only has peak values at the frequencies $f=825 \mathrm{~Hz}, f=1475 \mathrm{~Hz}$, and $f=1650 \mathrm{~Hz}$ in the frequency-domain characteristics of the bearing restraint force.

In summary, under this control strategy, the power coupling mechanism works smoothly and the time-domain and frequency-domain characteristics are sound during the mode switching process, which contributes to improving the vibration and noise of the vehicle.

\section{Conclusion}

In order to improve the ride comfort of a hybrid electric vehicle using double-row planetary gear power coupling 
mechanism during mode switching process, a dynamic coordinated control strategy of "torque distribution + engine torque monitoring + motor torque compensation" was proposed. The mathematical model and control strategy model were established; some simulation analyses were carried out in order to verify the proposed coordinated control strategy. The main conclusions can be summarized as follows:

(1) When switching from electric mode to joint driving mode is applied, the dynamic coordinated control strategy proposed in this study can effectively reduce the driving torque fluctuation and vehicle impact and improve the vehicle ride comfort. It is verified that the proposed dynamic coordinated control strategy is effective.

(2) The simulation analysis of the time-frequency characteristics of the gear meshing force and bearing restraint force indicates that the gear meshing force and bearing restraint force in the power coupling mechanism change smoothly and there is no obvious impact under the proposed control strategy, which is conducive to improve the vibration and noise characteristics of vehicles.

\section{Data Availability}

The data used to support the findings of this study are available from the corresponding author upon request.

\section{Conflicts of Interest}

The authors declare that there are no conflicts of interest regarding the publication of this paper.

\section{Acknowledgments}

This work was financially supported by the National Natural Science Foundation of China (51575238).

\section{References}

[1] S. H. Wang, C. R. He, H. Ren et al., "Study on power switching process of a hybrid electric vehicle with in-wheel motors," Shock and Vibration, vol. 2016, Article ID 7039309, 7 pages, 2016.

[2] H. S. Kim, J. H. Him, and H. C. Lee, "Mode transition control using disturbance compensation for a parallel hybrid electric vehicle," Proceedings of the Institution of Mechanical Engineers Part D-Journal of Automotive Engineering, vol. 2, no. 225, pp. 150-216, 2011.

[3] S. Kim, J. Park, J. Hong, M. Lee, and H. Sim, "Transient control strategy of hybrid electric vehicle during mode change," in Proceedings of the SAE World Congress o Exhibition, Detroit, MI, USA, April 2009.

[4] Y. B. Yan, F. W. Yan, and C. Q. Du, "Research on strategy and simulation for dynamic coordinative control of PHEV," China Mechanical Engineering, vol. 21, no. 2, pp. 234-239, 2010, in Chinese.

[5] W. H. Wang, W. K. Wang, B. Feng et al., "Dynamic coordinated control method of driving mode switch of parallel hybrid electric vehicle," Journal of Traffic and Transportation Engineering, vol. 17, no. 2, pp. 90-97, 2017, in Chinese.

[6] Z. G. Zhao, X. J. Dai, C. Wang et al., "Coordinated control of driving mode switching of compound power-split hybrid electric car," Automotive Engineering, vol. 37, no. 3, pp. 260-265, 2015, in Chinese.

[7] C. Liu, L. Chu, J. H. Guo et al., "Torque coordination algorithm for driving mode-transition of PHEV," Journal of Huazhong University of Science and Technology: Natural Sciences, vol. 41, no. 12, pp. 85-89, 2013, in Chinese.

[8] V. Parmar, R. D. Di, M. Sopouch et al., "Multiphysics simulation model for noise and vibration effects in hybrid vehicle powertrain," in Proceedings of the 8th International Styrian Noise, Vibration \& Harshness Congress: The European Automotive Noise Conference, June 2014.

[9] S. Tomura, Y. Ito, K. Kamichi et al., "Development of vibration reduction motor control for series-parallel hybrid system," in Proceedings of the SAE 2006 World Congress \& Exhibition, Detroit, MI, USA, April 2006.

[10] M. Yazaki, "Vibration reduction in motors for the sport hybrid SH-AWD," SAE International Journal of Alternative Powertrains, vol. 4, no. 1, pp. 153-161, 2015.

[11] R. I. Davis and R. D. Lorenz, "Engine torque ripple cancellation with an integrated starter alternator in a hybrid electric vehicle: implementation and control," IEEE Transactions on Industry Applications, vol. 39, no. 6, pp. 1765-1774, 2003.

[12] L. J. Xiao, M. Wang, Z. H. Zhong et al., "Coordinated shift control and experimental study of two-speed AMT for pure electric vehicle," Journal of Hunan University: Natural Sciences, vol. 46, no. 2, pp. 10-18, 2019, in Chinese.

[13] R. Beck, F. Richert, A. Bollig et al., "Model predictive control of a parallel hybrid vehicle drivetrain," in Proceedings of the 44th IEEE Conference on Decision and Control and the European Control Conference, pp. 2670-2675, Seville, Spain, December 2005.

[14] J. Sun, G. Xing, X. Liu, X. Fu, and C. Zhang, “A novel torque coordination control strategy of a single-shaft parallel hybrid electric vehicle based on model predictive control," Mathematical Problems in Engineering, vol. 2015, pp. 1-12, 2015.

[15] K. Koprubasi, E. R. Westervelt, and G. Rizzoni, "Toward the systematic design of controllers for smooth hybrid electric vehicle mode changes," in Proceedings of the 2007 American Control Conference, The American Automatic Control Council, New York, NY, USA, pp. 2985-2990, July 2007.

[16] W. K. Shi, P. F. Qin, Y. L. Yang et al., "Working mode analysis and parameter matching study of a novel power coupling system," Journal of Machine Design, vol. 33, no. 10, pp. 5-10, 2016, in Chinese.

[17] L. Chu, P. Z. Zhang, and T. T. Lin, "Study on mode recognition and mode switching strategy of electric vehicle," Journal of Huazhong University of Science and Technology: Natural Sciences, vol. 42, no. 6, pp. 12-16, 2014, in Chinese.

[18] X. Wei, P. Pisu, G. Rizzoni et al., "Dynamic modeling of a hybrid electric drivetrain for fuel economy, performance and driveability evaluations," in Proceedings of the ASME 2003 International Mechanical Engineering Congress and Exposition, pp. 443-450, Washington, DC, USA, November 2003.

[19] W. J. Zhou, Y. Y. Zuo, and M. Y. Zheng, "Analysis and optimization of the vibration and noise of a double planetary gear power coupling mechanism," Shock and Vibration, vol. 2018, Article ID 9048695, 2018.

[20] G. Zhu, J. Liu, J. Fu, Z. Xu, Q. Guo, and H. Zhao, "Experimental study on combustion and emission characteristics of turbocharged gasoline direct injection (GDI) engine under 
cold start new European driving cycle (NEDC)," Fuel, vol. 215, pp. 272-284, 2018.

[21] M. Y. Zheng, Y. Y. Zuo, C. G. Wu et al., "Dynamic characteristic analysis of double planetary gear power coupling mechanism," Journal of Chongqing University of Technology: Natural Sciences, vol. 31, no. 6, pp. 47-56, 2017, in Chinese. 